\title{
ON 4-MANIFOLDS WITH FINITELY DOMINATED COVERING SPACES
}

\author{
JONATHAN A. HILLMAN \\ (Communicated by Ronald Stern)
}

\begin{abstract}
We show that if the universal covering space $\widetilde{M}$ of a closed 4manifold $M$ is finitely dominated then either $M$ is aspherical, or $\tilde{M}$ is homotopy equivalent to $S^{2}$ or $S^{3}$, or $\pi_{1}(M)$ is finite. We also give a criterion for a closed 4-manifold to be homotopy equivalent to one which fibres over the circle.
\end{abstract}

\section{INTRODUCTION}

If $M$ is the total space of a fibration with finitely dominated base $B$ and fibre $F$ then $M$ is a Poincare duality complex if and only if both $B$ and $F$ are (cf. [Go]). In particular, if $p: M_{G} \rightarrow M$ is a regular covering of a closed manifold $M$ with covering group $G$ then $M_{G}$ is a Poincare complex if it is finitely dominated and $G$ is $F P$.

In this note we shall show that if $M$ is a 4-manifold the latter conditions may be relaxed considerably. (The corresponding results are trivial for surfaces and are easy for 3-manifolds.) In Theorem 1 we show that $M_{G}$ is a Poincaré complex if and only if it is finitely dominated and (if $G$ has one end) its fundamental group is $F P_{3}$. In Theorem 2 we give direct homological arguments to extend results of Hendriks and Laudenbach [HL] and Swarup [Sw] on the second homotopy groups of 3-manifolds to $P D_{3}$-complexes with torsion free fundamental group. These results are used in Theorem 3, which considers infinite cyclic covers of 4-manifolds (i.e., $G \cong Z$ ). Here we show that if $\pi_{1}\left(M_{G}\right)$ is virtually torsion free and is the fundamental group of a $P D_{3}$-complex and $\operatorname{Hom}_{Z[\pi]}\left(\pi_{2}(M), Z[\pi]\right)=0$ (where $\pi=\pi_{1}(M)$ ) then $M_{G}$ is a $P D_{3}$-complex.

Our arguments rest on Poincare duality and the Universal Coefficient spectral sequence (UCSS) for a regular covering $p: M_{G} \rightarrow M$ with covering group $G$. This spectral sequence has $E_{2}^{p q}$ term $\operatorname{Ext}_{Z[G]}^{q}\left(H_{p}\left(M_{G} ; Z\right), Z[G]\right)$ and converges to $H^{p+q}(M ; Z[G])$. Similarly, we shall let LHSSS denote the LyndonHochschild-Serre spectral sequence for an extension of groups. If $G$ is a group and $w: G \rightarrow\{ \pm 1\}$ is a homomorphism then $\bar{g}=w(g) g^{-1}$ defines an involution on $Z[G]$. If $L$ is a left $Z[G]-$ module then $\bar{L}$ shall denote the conjugate

Received by the editors September 1, 1992.

1991 Mathematics Subject Classification. Primary 57N13; Secondary 57P10.

Key words and phrases. Finitely dominated, 4-manifold, mapping torus, $P D_{3}$-complex, surface bundle. 
right $Z[G]$-module with the same underlying abelian group and $Z[G]$-action given by $l . g=\bar{g} . l$, for all $l \in L$ and $g \in G$. (We shall also use the overbar to denote the conjugate of a right $Z[G]$-module.) The conjugate of a free left (right) module is a free right (left) module of the same rank.

A group $G$ is $F P_{n}$ if the augmentation $Z[G]$-module $Z$ has a projective resolution which is finitely generated in degrees $\leq n$. It is $F P$ if it has finite cohomological dimension and is $F P_{n}$ for $n=$ c.d. $G$. "Finitely generated" is equivalent to $F P_{1}$, while "finitely presentable" implies $F P_{2}$. Groups which are $F P_{2}$ are also said to be almost finitely presentable.

A $P D_{n}$-complex is a Poincaré duality complex of formal dimension $n$, in other words, a finitely dominated cell complex which satisfies Poincare duality with arbitrary local coefficients. As such spaces are finitely dominated they have finitely presentable fundamental groups.

\section{FINITELY DOMINATED COVERING SPACES}

In this section we shall show that if a closed 4-manifold has an infinite regular covering space which is finitely dominated then the manifold is either aspherical or is close to being the total space of a fibre bundle.

Theorem 1. Let $M$ be a closed 4-manifold with fundamental group $\pi$, and let $\Pi=\pi_{2}(M)$. Suppose that $p: M_{G} \rightarrow M$ is a regular covering map, with covering group $G=\operatorname{Aut}(p)$ and such that $M_{G}$ is finitely dominated. Then

(i) $G$ has finitely many ends;

(ii) if $M_{G}$ is acyclic then it is contractible and $M$ is aspherical;

(iii) if $G$ has one end and $\pi_{1}\left(M_{G}\right)$ is infinite and $F P_{3}$ then $M$ is aspherical and $M_{G}$ is homotopy equivalent to $S^{1}$ or to an aspherical closed surface;

(iv) if $G$ has one end and $\pi_{1}\left(M_{G}\right)$ is finite but $M_{G}$ is not acyclic then $M_{G} \simeq S^{2}$ or $R P^{2}$

(v) $G$ has two ends if and only if $M_{G}$ is a $P D_{3}$-complex.

Proof. We may clearly assume that $G$ is infinite and $M$ is orientable. As $Z[G]$ has no nonzero (left) ideal (i.e., submodule) which is finitely generated as an abelian group $\operatorname{Hom}_{G}\left(H_{p}\left(M_{G} ; Z\right), Z[G]\right)=0$ for all $p \geq 0$, the bottom row of the UCSS for the covering $p$ is 0 . From the UCSS and Poincare duality we find that $H^{1}(G ; Z[G]) \cong \bar{H}_{3}\left(M_{G} ; Z\right)$. As this group is finitely generated and $G$ is infinite, $G$ has one or two ends.

Suppose that $G$ has one end. Then $H_{3}\left(M_{G} ; Z\right)=H_{4}\left(M_{G} ; Z\right)=0$. If $M_{G}$ is acyclic then $G$ is a $P D_{4}$-group, so $M_{G}$ is a $P D_{0}$-complex and, hence, contractible, by [Go]. Hence $M$ is aspherical.

If $\pi_{1}\left(M_{G}\right)$ is $F P_{3}$ then $\Pi=\pi_{2}(M)=\pi_{2}\left(M_{G}\right)$ is finitely generated as a module over $Z\left[\pi_{1}\left(M_{G}\right)\right]$, so $\operatorname{Hom}_{\pi}(\Pi, Z[\pi])=0$. If, moreover, $\pi_{1}\left(M_{G}\right)$ is infinite then $H^{s}(\pi ; Z[\pi])=0$ for $s \leq 2$. Hence $\Pi=0$, by the UCSS and Poincare duality, so $M$ is aspherical. We may then apply an LHSSS corner argument to show that if $H^{2}(G ; Z[G]) \neq 0$ then $\pi_{1}\left(M_{G}\right)$ has one end and $H^{2}\left(\pi_{1}\left(M_{G}\right) ; Z\left[\pi_{1}\left(M_{G}\right)\right]\right) \cong Z$. Since $\pi_{1}\left(M_{G}\right)$ is $F P$, it is a $P D_{2}$-group by [Fa2, Theorem 3], so $M_{G}$ is homotopy equivalent to an aspherical closed surface. If $H^{2}(G ; Z[G])=0$ then $H^{1}\left(\pi_{1}\left(M_{G}\right) ; Z\left[\pi_{1}\left(M_{G}\right)\right]\right) \cong Z$ by another LHSSS corner argument. Since $\pi_{1}\left(M_{G}\right)$ is $F P$ and hence torsionfree, it must be infinite cyclic. Therefore, $M_{G} \simeq S^{1}$. 
If $\pi_{1}\left(M_{G}\right)$ is finite but $M_{G}$ is not acyclic then the universal covering space $\tilde{M}$ is also finitely dominated but not contractible and $\Pi=H_{2}(\tilde{M} ; Z)$ is a nontrivial finitely generated abelian group, while $H_{3}(\tilde{M} ; Z)=H_{4}(\tilde{M} ; Z)=0$. Moreover, $H^{2}(\pi ; Z[\pi]) \cong \bar{\Pi}$ by the UCSS and Poincaré duality. Let $C$ be a finite cyclic subgroup of $\pi$ which acts trivially on $\Pi$. Then it follows easily from the Cartan-Leray spectral sequence for the projection of $\tilde{M}$ onto $\tilde{M} / C$ that there are isomorphisms $H_{n+3}(C ; Z) \cong H_{n}(C ; \Pi)$ for all $n>2$. If $n$ is odd then this isomorphism reduces to $0=\Pi /|C| \Pi$. Since $\Pi$ is finitely generated, this implies that multiplication by $|C|$ is an isomorphism. On the other hand, if $n$ is even then we have $Z /|C| Z \cong\{a \in \Pi:|C| a=0\}$. Hence we must have $C=1$. Now since $\Pi$ is finitely generated, any torsion subgroup of $\operatorname{Aut}(\Pi)$ is finite. (Let $T$ be the torsion subgroup of $\Pi$, and suppose that $\Pi / T \cong Z^{r}$. Then the natural homomorphism from $\operatorname{Aut}(\Pi)$ to $\operatorname{Aut}(\Pi / T)$ has finite kernel, and its image is isomorphic to a subgroup of $\operatorname{GL}(r, Z)$, which is virtually torsion free.) Hence as $\pi$ is infinite, it must have elements of infinite order, so $H^{2}(\pi ; Z[\pi])$ must be infinite cyclic by [Fa1, Corollary 5.2], as it is a nontrivial finitely generated group. Hence $\tilde{M} \simeq S^{2}$ and $\pi_{1}\left(M_{G}\right)$ has order at most 2 , so $M_{G} \simeq S^{2}$ or $R P^{2}$.

Suppose now that $G$ has two ends. We may assume that $G \cong Z$, and it then follows easily from [Go] that $M_{G}$ is a $P D_{3}$-complex. Conversely, if $M_{G}$ is a $P D_{3}$-complex then $G$ is infinite and $H^{1}(G ; Z[G]) \cong H_{3}\left(M_{G} ; Z\right)$, so $G$ has two ends.

Is the hypothesis in (iii) that $\pi_{1}\left(M_{G}\right)$ be $F P_{3}$ redundant?

Corollary 1. The covering space $M_{G}$ is homotopy equivalent to a closed surface if and only if it is finitely dominated, $G$ has one end, $H^{2}(G ; Z[G]) \cong Z$, and $\pi_{1}\left(M_{G}\right)$ is $F P_{3}$. The group $G$ is then $F P_{\infty}$. If, moreover, $G$ has a torsion free subgroup of finite index with infinite abelianization then $M$ has a finite covering space which is simple homotopy equivalent to a closed 4-manifold which fibres over an aspherical closed surface.

Proof. If $M_{G}$ is homotopy equivalent to a closed surface then it is finitely dominated, $\pi_{1}\left(M_{G}\right)$ is $F P_{3}$, and the conditions on $G$ in the first assertion follow from the UCSS. The converse follows from parts (iii) and (iv) of the theorem.

If $\tilde{M} \simeq S^{2}$ and $\pi$ is $F P_{n}$, we may splice together a cellular chain complex for $\tilde{M}$ and a finite partial resolution of the augmentation module $Z$ to show that $\pi$ is $F P_{n+3}$. (Since $M$ is homotopy equivalent to a finite cell complex, the singular chain complex of $\tilde{M}$ is chain homotopy equivalent to a finite free $Z[\pi]$-complex $C_{*}$. Let $P_{*}$ be a projective resolution of the augmentation module $Z$ in which $P_{0}=Z[\pi]$ and $P_{i}$ is finitely generated for all $i \leq n$. Let $Q_{n}=C_{n}$ if $n \leq 2$ and $Q_{n}=C_{n} \oplus P_{n-3}$ if $n \geq 3$, where the differential from $Q_{3}$ to $Q_{2}$ maps a generator of the summand $P_{0}$ onto a 2-cycle representing the generator of $H_{2}(\tilde{M} ; Z)$.) An induction on $n$ then shows that $\pi$ is $F P_{\infty}$. If $M$ is aspherical then $\pi$ is a $P D_{4}$-group and so is $F P$. Since finite groups are $F P_{\infty}$ and surface groups are $F P$, it follows that $\pi$ and $\pi_{1}\left(M_{G}\right)$ are both $F P_{\infty}$ in all cases. Hence, so is $G \cong \pi / \pi_{1}\left(M_{G}\right)$ by [B, Proposition 2.7].

Suppose finally that $H$ is a subgroup of finite index in $G$ which is torsion free and has infinite abelianization. Then $H$ is also finitely presentable, and 
$H^{2}(H ; Z[H]) \cong Z$. Therefore, $H$ has finite cohomological dimension by [Fa1, Theorem 2.3] and so is a $P D_{2}$-group by [Fa2]. The finite covering space $M_{1}=$ $M_{G} / H$ is simple homotopy equivalent to the total space of an $M_{G}$-bundle over the aspherical closed surface with fundamental group $H$ by [Hi3, Theorems 1 , 3 , and 4].

Does $H^{2}(G ; Z[G]) \cong Z$ always imply that $G$ is virtually a surface group? Is $M$ in fact finitely covered by such a bundle space?

Corollary 2. The covering space $M_{G}$ is homotopy equivalent to $S^{1}$ if and only if it is finitely dominated, $G$ has one end, $H^{2}(G ; Z[G])=0$ and $\pi_{1}\left(M_{G}\right)$ is a nontrivial finitely generated free group. Moreover, $M$ is homotopy equivalent to the total space of an $S^{1}$-bundle over a $P D_{3}$-complex if and only if $G$ has finite cohomological dimension.

Proof. If $M_{G} \simeq S^{1}$ then it is finitely dominated, $M$ is aspherical, and the conditions on $G$ in the first assertion follow from the LHSSS. The converse follows from part (iii) of the theorem, since a nontrivial finitely generated free group is infinite and $F P$. The second assertion follows from [Hi4].

Corollary 3. If the universal covering space $\tilde{M}$ is finitely dominated then either $M$ is aspherical, or $\tilde{M}$ is homotopy equivalent to $S^{2}$ or $S^{3}$, or $\pi_{1}(M)$ is finite.

If $\tilde{M}$ is homotopy equivalent to $S^{2}$ and $\pi$ has a subgroup of finite index with infinite abelianization then $\tilde{M}$ is homeomorphic to $S^{2} \times R^{2}$ [Hi5].

We may ask how far the results of this section may be extended to arbitrary coverings. If $p: \hat{M} \rightarrow M$ is an infinite covering with $\hat{M}$ finitely dominated then must we have either $M$ is aspherical, or $\tilde{M}$ is homotopy equivalent to $S^{2}$, or $\hat{M}$ is a $P D_{3}$-complex and $\left[\pi: N_{\pi}(\hat{\pi})\right]$ is finite?

\section{The Algebraic 2-type of A $P D_{3}$-COMPleX}

In this section we shall use Schanuel's lemma to obtain some information on the algebraic 2-type of a $P D_{3}$-complex with torsion free fundamental group, which we shall use in the next section. (We prove more than we need.)

Theorem 2. Let $N$ be a $P D_{3}$-complex with torsionfree fundamental group $\nu$. Then

(i) $\pi_{2}(N)$ is finitely generated as a $Z[\nu]$-module;

(ii) if $\nu$ is a nontrivial free group then $H^{2}\left(N ; \pi_{2}(N)\right) \cong Z$;

(iii) if $\nu$ is not free then $\pi_{2}(N)$ is a projective $Z[\nu]$-module and $H^{2}\left(N ; \pi_{2}(N)\right)$ $=0$;

(iv) if $\nu$ is not free any two of the conditions " $\nu$ is $F F ", " N$ is homotopy equivalent to a finite complex", and " $\pi_{2}(N)$ is stably free" imply the third.

Proof. We may clearly assume that $\nu \neq 1$. The $P D_{3}$-complex $N$ is homotopy equivalent to a connected sum of aspherical $P D_{3}$-complexes and a 3-manifold with free fundamental group by [Tu, Theorem 2]. Therefore, $\nu$ is a corresponding free product, so it has cohomological dimension at most 3 and is $F P$.

Since $N$ is finitely dominated, the equivariant chain complex of the universal covering space $\tilde{N}$ is chain homotopy equivalent to a complex $0 \rightarrow C_{3} \rightarrow C_{2} \rightarrow$ $C_{1} \rightarrow C_{0} \rightarrow 0$ of finitely generated projective left $Z[\nu]$-modules. Then the sequences $0 \rightarrow Z_{2} \rightarrow C_{2} \rightarrow C_{1} \rightarrow C_{0} \rightarrow Z \rightarrow 0$ and $0 \rightarrow C_{3} \rightarrow Z_{2} \rightarrow$ $\pi_{2}(N) \rightarrow 0$ are exact, where $Z_{2}$ is the module of 2-cycles in $C_{2}$. Since $\nu$ 
is $F P$ and c.d. $\nu \leq 3$, Schanuel's lemma implies that $Z_{2}$ is projective and finitely generated. Hence $\pi_{2}(N)$ has projective dimension at most 1 and is finitely generated.

It follows easily from the UCSS and Poincare duality that $\pi_{2}(N) \cong$ $\bar{H}^{1}(\nu ; Z[\nu])$ and that there is an exact sequence

$$
H^{3}(\nu ; Z[\nu]) \rightarrow H^{3}(N ; Z[\nu]) \rightarrow \operatorname{Ext}_{\nu}^{1}\left(\pi_{2}(N), Z[\nu]\right) \rightarrow 0 .
$$

The $w_{1}(N)$-twisted augmentation homomorphism from $Z[\nu]$ to $\bar{Z}$ which sends $g \in \nu$ to $w_{1}(N)(g)$ induces an isomorphism from $H^{3}(N ; Z[\nu])$ to $H^{3}(N ; \bar{Z}) \cong Z$.

If $\nu$ is free then the first term in this sequence is 0 , so $\operatorname{Ext}_{\nu}^{1}\left(\pi_{2}(N), Z[\nu]\right) \cong$ $Z$. (In particular, $\pi_{2}(N)$ has projective dimension 1.) There is also a short exact sequence of left modules $0 \rightarrow Z[\nu]^{r} \rightarrow Z[\nu] \rightarrow Z \rightarrow 0$, where $r$ is the rank of $\nu$. On dualizing we obtain the sequence of right modules $0 \rightarrow Z[\nu] \rightarrow$ $Z[\nu]^{r} \rightarrow H^{1}(\nu ; Z[\nu]) \rightarrow 0$. The long exact sequence of homology with these coefficients includes an exact sequence

$$
\begin{aligned}
0 & \rightarrow H_{1}\left(N ; H^{1}(\nu ; Z[\nu])\right) \rightarrow H_{0}(N ; Z[\nu]) \\
& \rightarrow H_{0}\left(N ; Z[\nu]^{r}\right) \rightarrow H_{0}\left(N ; H^{1}(\nu ; Z[\nu])\right),
\end{aligned}
$$

in which the middle map is 0 , so $H_{1}\left(N ; H^{1}(\nu ; Z[\nu])\right) \cong H_{0}(N ; Z[\nu])=Z$. Poincaré duality then gives

$$
H^{2}\left(N ; \pi_{2}(N)\right) \cong H_{1}\left(N ; \bar{\pi}_{2}(N)\right)=H_{1}\left(N ; H^{1}(\nu ; Z[\nu])\right) \cong Z \text {. }
$$

If $\nu$ is not free then the map $H^{3}(\nu ; Z[\nu]) \rightarrow H^{3}(N ; Z[\nu])$ in the above sequence is onto, as can be seen by comparison with the corresponding sequence with coefficients $\bar{Z}$. Therefore, $\operatorname{Ext}_{\nu}^{1}\left(\pi_{2}(N), Z[\nu]\right)=0$. Since $\pi_{2}(N)$ has projective dimension at most one, it follows that $\pi_{2}(N)$ is in fact projective. As $H^{2}(N ; Z[\nu])=H_{1}(N ; Z[\nu])=0$, it follows that $H^{2}(N ; P)=0$ for any projective $Z[\nu]$-module $P$. Therefore, $H^{2}\left(N ; \pi_{2}(N)\right)=0$.

The final assertion follows easily from the fact that if $\pi_{2}(N)$ is projective then $Z_{2} \cong \pi_{2}(N) \oplus C_{3}$.

If $\nu$ is not torsion free then the projective dimension of $\pi_{2}(N)$ is infinite. Swarup showed that if $N$ is a 3-manifold which is the connected sum of a 3manifold whose fundamental group is free of rank $r$ with $s \geq 1$ aspherical 3manifolds then $\pi_{2}(N)$ is a finitely generated free $Z[\nu]$-module of rank $2 r+s-1$ [Sw].

The cohomology group $H^{2}\left(N ; \pi_{2}(N)\right)$ arises in studying homotopy classes of self-homotopy equivalences of $N$. Hendriks and Laudenbach [HL] show that if $N$ is a $P^{2}$-irreducible 3 -manifold and $\pi_{1}(N)$ is virtually free then $H^{2}\left(N ; \pi_{2}(N)\right) \cong Z$; otherwise $H^{2}\left(N ; \pi_{2}(N)\right)=0$. Does this result extend to $P D_{3}$-complexes with virtually torsion free fundamental group?

\section{MAPPING TORI}

An infinite cyclic covering space of a closed $n$-manifold $M$ is homotopy equivalent to a $P D_{n-1}$-complex if and only if it is finitely dominated. The fundamental group $\nu$ of the covering space must then be finitely generated and $\chi(M)=0$. It is conceivable that these conditions may suffice when $n=4$; this is also so if either $\nu$ is free or $\nu$ does not contain the centre of $\pi_{1}(M)$ [Hi1]. Here we shall give another criterion. 
Theorem 3. Let $M$ be a closed 4-manifold whose fundamental group $\pi$ is an extension of $Z$ by a virtually torsion free normal subgroup $\nu$, and let $\Pi=$ $\pi_{2}(M)$. Then the infinite cyclic covering space $M^{\prime}$ with fundamental group $\nu$ is homotopy equivalent to a $P D_{3}$-complex if and only if $\nu$ is the fundamental group of a $P D_{3}$-complex $N, \Pi^{*}=\operatorname{Hom}_{\pi}(\Pi, Z[\pi])$ is 0 and the images of $k_{1}(M)$ and $k_{1}(N)$ in $H^{3}\left(\nu_{0} ; \pi_{2}(M)\right) \cong H^{3}\left(\nu_{0} ; \pi_{2}(N)\right)$ generate the same subgroup under the action of $\operatorname{Aut}_{Z\left[\nu_{0}\right]}\left(\pi_{2}(N)\right)$, where $\nu_{0}$ is a torsion free subgroup of finite index in $\nu$. If, moreover, $\nu$ is torsion free and $\left.w_{1}(M)\right|_{\nu}=w_{1}(N)$ then $M^{\prime} \simeq N$, and if $N$ is a 3-manifold whose irreducible factors are Haken, hyperbolic, or Seifert-fibred then $M$ is homotopy equivalent to the total space of an $N$-bundle over $S^{1}$.

Proof. Suppose first that $\nu$ is torsion free and $\left.w_{1}(M)\right|_{\nu}=w_{1}(N)$. If $\nu=1$ then $M^{\prime}$ is simply connected and $\pi \cong Z$ has two ends. It follows immediately from the UCSS and Poincaré duality that $H_{2}\left(M^{\prime} ; Z\right)=\Pi \cong \bar{\Pi}^{*}$ and $H_{3}\left(M^{\prime} ; Z\right) \cong Z$. Therefore, $M^{\prime}$ is a $P D_{3}$-complex if and only if $\Pi^{*}=0$, and it is then homotopy equivalent to $S^{3}$.

If $\nu$ is torsion free but nontrivial then $\pi$ has one end and $N$ is homotopy equivalent to a connected sum of aspherical $P D_{3}$-complexes and a 3-manifold with free fundamental group [Tu]. Therefore, c.d. $\nu \leq 3$ and c.d. $\pi \leq 4$. It follows immediately from the UCSS that $e_{\pi}^{s}(\Pi)=0$ if $s>2$. Thus $\Pi$ has projective dimension at most 2 as a $Z[\pi]$-module and a fortiori as a $Z[\nu]$ module. Hence $H^{3}(\nu ; \Pi)=0$. Moreover, the UCSS and Poincaré duality together with the hypothesis that $\Pi^{*}=0$ imply that $\Pi \cong \bar{H}^{2}(\pi ; Z[\pi])$. Hence $\Pi \cong \bar{H}^{1}(\nu ; Z[\nu])$ by the LHSSS. (The overbar notation is unambiguous since $\left.w_{1}(M)\right|_{\nu}=w_{1}(N)$.) But this is isomorphic to $\pi_{2}(N)$ by Poincaré duality for $N$. Since the first $k$-invariants of $M^{\prime}$ and $N$ are equivalent, these spaces have the same algebraic 3-type. Since $N$ is homotopy equivalent to a 3-dimensional complex, there is a map $f: N \rightarrow M^{\prime}$ which induces isomorphisms on fundamental group and second homotopy group. Since the homology of the universal covering spaces of these spaces vanishes above degree 2 , the map $f$ is a homotopy equivalence.

Conversely, if $M^{\prime}$ is homotopy equivalent to $N$ then $\Pi \cong \bar{H}^{1}(\nu ; Z[\nu])$ is finitely generated as a $Z[\nu]$-module. As 0 is the only $Z[\pi]$-submodule of $Z[\pi]$ which is finitely generated as a $Z[\nu]$-module, it follows that $\Pi^{*}=0$.

In general, let $\nu_{o} \subseteq \nu_{+} \cap \pi_{+}$be a torsion free subgroup of finite index in $\nu$, where $\pi_{+}=\operatorname{ker} w_{1}(M)$ and $\nu_{+}=\operatorname{ker} w_{1}(N)$, and let $t \in \pi$ generate $\pi$ modulo $\nu$. Then each of the conjugates $t^{k} \nu_{o} t^{-k}$ in $\pi$ has the same index in $\nu$. Since $\nu$ is finitely generated, the intersection $\mu=\bigcap t^{k} \nu_{o} t^{-k}$ of all such conjugates has finite index in $\nu$ and is clearly torsion free and normal in the subgroup $\rho$ generated by $\mu$ and $t$. It is easily seen that $M^{\prime}$ is a $P D_{3}$-complex if and only if the covering space with fundamental group $\mu$ is. Moreover, $\operatorname{Hom}_{\rho}(\Pi, Z[\rho])=0$ if and only if $\Pi^{*}=0$. (For if $\left\{r_{i}\right\}$ is a transversal for $\rho$ in $\pi$ and $f: \Pi \rightarrow Z[\rho]$ is a nontrivial $Z[\rho]$-linear homomorphism then $g(m)=\sum r_{i} f\left(r_{i}^{-1} m\right)$ defines a nontrivial element of $\Pi^{*}$. The converse is clear.)

Now suppose that there is such a homotopy equivalence $f: N \rightarrow M^{\prime}$, where $N$ is a 3-manifold whose irreducible factors are as above. Let $t: M^{\prime} \rightarrow M^{\prime}$ be the generator of the covering transformations. Then there is a self-homotopy 
equivalence $u: N \rightarrow N$ such that $f u \sim t f$. As each irreducible factor of $N$ has the property that self-homotopy equivalences are homotopic to homeomorphisms [Mo, Sc, Wd], $u$ is homotopic to a homeomorphism [HL], so $M$ is homotopy equivalent to the mapping torus of this homeomorphism.

All known $P D_{3}$-complexes are homotopy equivalent to connected sums of Haken, hyperbolic or Seifert-fibred 3-manifolds, and $P D_{3}$-complexes with finite fundamental group and, in particular, have virtually torsion free fundamental group.

The condition $\Pi^{*}=0$ is often implied by the simpler condition $\chi(M)=0$. For instance, this is so if $\nu$ is free (see [H, Chapter VII, Theorem 4]), so Theorem 3 gives an alternative proof of [Hi1, Theorem 1]. The condition $\chi(M)=0$ also implies $\Pi^{*}=0$ if $\pi$ is amenable or $Z[\pi]$ embeds in a ring with the SIBN property which is flat as a $Z[\pi]$-module and in which an element of the augmentation ideal becomes invertible, by [Ec] and [Hi2], respectively. (A ring $R$ has the SIBN (stably invariant basis number) property if nontrivial stably free $R$-modules have well-defined, strictly positive rank-in other words, if whenever an $R$-module $L$ satisfies $L \oplus R^{a} \cong R^{b}$ for some nonnegative integers $a, b$, then $b-a$ depends only on $L$ and is 0 if and only if $L=0$.)

It would also be of interest to replace the condition that $\nu$ be isomorphic to the group of a $P D_{3}$-complex by more intrinsic, algebraic conditions. For instance, if $\chi(M)=0, \pi$ has an infinite cyclic normal subgroup, and $\nu$ is $F P_{2}$ then $M^{\prime}$ is a $P D_{3}$-complex. (Note that here we do not assume a priori that $\nu$ is the fundamental group of a $P D_{3}$-complex). This is [Hi1, Theorem 2] if the cyclic normal subgroup is not contained in $\nu$ and follows from the argument of [H, Chapter IV, Theorem 5] otherwise.

\section{REFERENCES}

[B] R. Bieri, Homological dimension of discrete groups, Queen Mary College Lecture Notes, London, 1976.

[Ec] B. Eckmann, Manifolds of even dimension with amenable fundamental group, preprint, ETH Zürich, February, 1993.

[Fa1] F. T. Farrell, The second cohomology group of $G$ with coefficients $Z / 2 Z[G]$, Topology 13 (1974), 313-326.

[Fa2] - Poincaré duality and groups of type FP, Comment. Math. Helv. 50 (1975), 187-195.

[Go] D. H. Gottlieb, Poincaré duality and fibrations, Proc. Amer. Math. Soc. 76 (1979), 148-150.

[HL] H. Hendriks and F. Laudenbach, Scindement d'une équivalence d'homotopie en dimension 3, Ann. Sci. École Norm. Sup. (4) 7 (1974), 203-217.

[H] J. A. Hillman, 2-knots and their groups, Austral. Math. Soc. Lect. Ser., vol. 5, Cambridge Univ. Press, Cambridge and New York, 1989.

[Hil] _ A homotopy fibration theorem in dimension four, Topology Appl. 33 (1989), 151-161.

[Hi2] __ Elementary amenable groups and 4-manifolds with Euler characteristic 0, J. Austral. Math. Soc. 50 (1991), 160-170.

[Hi3] _ On 4-manifolds homotopy equivalent to surface bundles over surfaces, Topology Appl. 40 (1991), 275-286.

[Hi4] , On 4-manifolds homotopy equivalent to circle bundles over 3-manifolds, Israel J. Math. 75 (1991), 277-287.

[Hi5] _ On 4-manifolds with universal covering space $S^{2} \times R^{2}$ or $S^{3} \times R$, Topology Appl. 52 (1993), 23-42. 
[Mo] G. D. Mostow, Quasi-conformal mappings in $n$-space and the rigidity of hyperbolic space forms, Inst. Hautes Études Sci. Publ. Math. 34 (1968), 53-104.

[Sc] G. P. Scott, There are no fake Seifert fibre spaces with infinite $\pi_{1}$, Ann. of Math. (2) 117 (1983), 35-70.

[Sw] G. A. Swarup, On embedded spheres in 3-manifolds, Math. Ann. 203 (1973), 89-102.

[Tu] V. G. Turaev, Three-dimensional Poincaré complexes: homotopy classification and splitting, Math. USSR Sb. 67 (1990), 261-282.

[Wd] F. Waldhausen, On irreducible 3-manifolds which are sufficiently large, Ann. of Math. 87 (1968), 56-88.

[Wl] C. T. C. Wall, Finiteness conditions for CW-complexes. I, Ann. of Math. (2) 81 (1965), 56-69.

School of Mathematics and Statistics, The University of Sydney, Sydney, New South WALES 2006, Australia

E-mail address: hillman_j@maths . su .oz . au 